\title{
Prosthetic Valve Dysfunction
}

National Cancer Institute

\section{Source}

National Cancer Institute. Prosthetic Valve Dysfunction. NCI Thesaurus. Code C111650.

Malfunction of a surgically-placed cardiac valve related to the valve itself or the area around the valve. 